\title{
COUNTING WORD-TREES
}

\author{
by J. M. O. MITCHELL
}

(Received 23 August, 1973)

Introduction. In his recent study of free inverse semigroups, Munn [2] introduced and used extensively the concept of a word-tree. In this note the number of such trees is found.

Definition. A word-tree on an alphabet $A$ is a finite tree, with at least two points, that satisfies the following conditions.

(WT1) Each line is oriented and is labelled by an element of $A$.

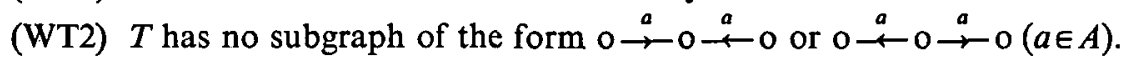

Lines will be described by an ordered pair of adjacent points and are said to be similar if their orientations and labellings are the same.

A line $\alpha \beta$, where $\beta$ is an endpoint of a word-tree $T$, is called an endline of $T$.

Isomorphism is defined in the obvious way (preserving orientation and labelling of lines).

A fundamental fact proved in Munn's paper [2, Theorem 2.2] is that a word-tree has no nontrivial automorphism.

Notations. $\quad d(\alpha)=$ degree of point $\alpha$.

$k=$ cardinal of $A$, taken to be finite.

$n(p)=$ number of word-trees on $A$ with $p$ points.

$n(p, e)=$ number of word-trees on $A$ with $p$ points and $e$ endpoints.

$(n)_{j}= \begin{cases}n(n-1) \ldots(n-j+1) & (j=1,2, \ldots), \\ 1 & (j=0) .\end{cases}$

Note that $n(p, e)=0$ if $e<2$ or $p<e$ and that, for $p>2, n(p, p)=0$.

Construction. Given a word-tree $T$ with $p-1$ points and $e$ endpoints, we can construct a larger one by adding a new line at any point. The number of dissimilar lines that may be added at $\alpha$ is $2 k-d(\alpha)$, since each old line terminating in $\alpha$ imposes one restriction on the new line and, by (WT2), these restrictions are different. The new word-tree has $e$ endpoints if the new line was added at an old endpoint and $e+1$ endpoints otherwise.

Let $T_{1}$ and $T_{2}$ be the word-trees obtained by adding endlines $\alpha_{1} \beta_{1}$ and $\alpha_{2} \beta_{2}$ respectively to $T$, where $\alpha_{1}$ and $\alpha_{2}$ are distinct points of $T$. Then, since $T$ has no nontrivial automorphisms, there can be no isomorphism $\phi: T_{1} \rightarrow T_{2}$ such that $\beta_{1} \phi=\beta_{2}$. Clearly the same result holds if $\alpha_{1}=\alpha_{2}$ but the endlines $\alpha_{1} \beta_{1}$ and $\alpha_{2} \beta_{2}$ are dissimilar. It follows that there is a $1-1$ correspondence between the possible constructions on word-trees with $p-1$ points and the wordtrees on $p$ points with a distinguished endpoint.

Calculation. From the tree $T$ the construction gives $(2 k-1) e$ word-trees with $e$ endpoints and $\sum_{\alpha}(2 k-d(\alpha))$ with $e+1$ endpoints, where the sum is over all points $\alpha$ of $T$ that are not 
endpoints of $T$. But $\sum_{\alpha} d(\alpha)+e=2$ (number of lines in $T$ ) $=2(p-2)$ (see, e.g., [1, Theorem 4.11]), so that the number of word-trees with $e+1$ endpoints is

$$
2 k(p-1-e)-2(p-2)+e \text {. }
$$

Among all possible constructions, a particular tree with $p$ points and $e$ points will occur $e$ times. Thus

$$
\begin{aligned}
n(p, e) & =\frac{1}{e}\{(2 k-1) \operatorname{en}(p-1, e)+(2 k(p-1-e+1)-2(p-2)+e-1) n(p-1, e-1)\} \\
& =(2 k-1) n(p-1, e)+\left\{\frac{2 k p-2 p+3}{e}-(2 k-1)\right\} n(p-1, e-1), \text { for } p \geqq 3 .
\end{aligned}
$$

This recurrence formula is used $p-3$ times to express $n(p)$, where $p \geqq 3$, in terms of $n(3,2)$, which is $k(2 k-1)$. In fact, for $0 \leqq j \leqq p-3$,

$$
n(p)=(2 k p-2 p+j+2)_{j} \sum_{e=2}^{p-j-1} n(p-j, e) /(e+j)_{j} .
$$

This is proved by induction on $j$. Application of (1) in (2) gives

$$
\begin{aligned}
n(p)=(2 k p-2 p+j+2)_{j} \sum_{e=2}^{p-j-1} & {[(2 k-1) n(p-j-1, e)+} \\
+ & \left.\left\{\frac{2 k(p-j)-2 p+2 j+3}{e}-(2 k-1)\right\} n(p-j-1, e-1)\right] /(e+j)_{j} .
\end{aligned}
$$

In the sum, the coefficient of $n(p-j-1, e)$, when $2 \leqq e \leqq p-j-2$, is

$$
\frac{2 k-1}{(e+j)_{j}}+\frac{2 k(p-j)-2 p+2 j+3}{(e+1)(e+1+j)_{j}}-\frac{2 k-1}{(e+1+j)_{j}}=(2 k p-2 p+j+3) /(e+j+1)_{j+1} \text {. }
$$

The other substitute terms are 0 . This verifies (2).

In particular, for $j=p-3$, we obtain

$$
\begin{aligned}
n(p) & =(2 k p-2 p+p-1)_{p-3} \sum_{e=2}^{2} n(3, e) /(e+p-3)_{p-3} \\
& =(2 k p-p-1)_{p-3} k(2 k-1) / \frac{(p-1) !}{2} \\
& =\frac{2 k(2 k-1)}{(p-1)(p-2)}\left(\begin{array}{c}
2 k p-p-1 \\
p-3
\end{array}\right) .
\end{aligned}
$$

ACKNowledgement. I am grateful to Professor Munn for showing me this problem and for letting me see his paper.

\section{REFERENCES}

1. F. Harary, Graph theory (Reading, Mass., 1969).

2. W. D. Munn, Free inverse semigroups, Proc. London Math. Soc.; to appear.

DePaRTMENT OF MATHEMATICS

UNIVERSITY OF STIRLING 